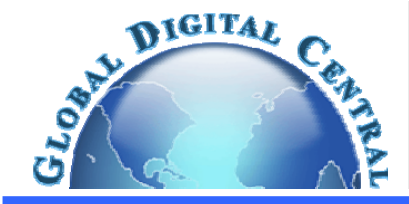

\title{
MEASUREMENTS OF THERMAL FIELD AT STACK EXTREMITIES OF A STANDING WAVE THERMOACOUSTIC HEAT PUMP
}

\author{
Syeda Humaira Tasnim ${ }^{\mathrm{a},}$, Shohel Mahmud ${ }^{\mathrm{b}}$, and Roydon Andrew Fraser ${ }^{\mathrm{a}}$ \\ ${ }^{a}$ Department of Mechanical and Mechatronics Engineering, University of Waterloo, Waterloo, ON, Canada N2L 3G1 \\ ${ }^{b}$ School of Engineering, University of Guelph, Guelph, ON, Canada N1G 2W1
}

\begin{abstract}
In this paper, we experimentally measure the temperature fields at different locations on the stack plate and in the surrounding working fluid in a standing wave thermoacoustic device. The temperature measurements at the stack extremities and at the neighboring gas show axial heat transfer at the stack extremities, as opposed to the hypothesis of a perfectly isolated stack used in the linear thermoacoustic theory. Four different mechanisms of heat transfer are identified at the stack extremities in the present study. This information is necessary for the optimization of the performances of practical thermoacoustic engines. For the selected operating conditions, temperature measurements along the stack reveal a linear behavior along the stack and the corresponding gas residing near the stack. Temperature gradient along the working fluid is higher than that of along the stack, as opposed to the equal temperature gradient assumed by the linear theory. This information might be incorporated into the linear thermoacoustic theory to accurately predict the temperature difference generated across the stack ends.
\end{abstract}

Keywords: Thermoacoustic heat pump; Thermal field measurement

\section{INTRODUCTION}

The thermal interaction of the acoustic waves with the stack creates an additional phase shift between the oscillating particle velocity and temperature in the standing acoustic wave, when the stack is placed in a quarter wavelength resonator driven by a loudspeaker (see Fig.1). This phase shift results in a longitudinal heat flux along the stack and in heating in one extremities and cooling of the other. Installation of heat exchangers near the extremities of the stack provides an opportunity to supply this heat flux inside the resonator (through the cold heat exchanger) and extract the heat flux from the resonator (through the hot heat exchanger). Thus a thermoacoustic heat pump can be used to heat the other systems which are connected to the hot heat exchanger.

Advantages of thermoacoustic devices include environmental friendliness, potentially high reliability due to simple structure and minimum number of moving parts, and reasonable efficiency. These characteristics could lead to low manufacturing and maintenance costs. Thermoacoustic devices exhibit their potential application in several areas: electronic cooling (high frequency piezoelectric cooler), low-temperature application (e.g., Ben \& Jerry's environment friendly cooler), remote space application (e.g., NASA's space shuttle), cryogenic application (e.g., ThermoacousticStirling system), refrigeration (e.g., solar powered thermoacoustic refrigerator, thermoacoustic refrigerator powered by waste heat), and hybrid system (e.g., thermoelectric-thermoacoustic cooling system).

The basic problem concerning a thermoacoustic device is the description of the temperature field and the heat flux distributions inside and at the edges of the stacks. This information is necessary for the optimization of the performances of practical thermoacoustic engines. The present work is a contribution to the study of temperature fields inside and at the edges of the stacks and at the neighboring working fluid, with an objective to improve the understanding of the physics underlying the thermoacoustic effect (longitudinal heat flux along the stack).

Several authors (Atchley et al. (1990), Worlikar et al. (1998), and Marx et al. (2005)) have made a study of temperature difference at the two stack extremities either experimentally or numerically and have made comparisons with the linear theory predictions (Swift (1988)). While a thermoacoustic device has to be designed, the major theory to rely upon is the linear theory (Swift (1988)). Piccolo and Cannistrato (2002) have proposed a simple calculus procedure based on the linear thermoacoustic theory to investigate the origin of the deviations of the predictions of the linear theory from the measured performances of real devices. The temperature differences measured in steady states by the authors do not match the linear theory predictions even at low Mach numbers, and discrepancies up to forty percent are measured. The calculus procedure proposed by the authors to calculate the convective heat flux in the early stage of transient regime indicates that the thermoacoustic heat flux carried by the stack as proposed by the linear theory is well predicted for that regime. Their results show that the reasons for the discrepancy regarding the steady state temperature difference might be the time average heat transfer processes not taken into account by the standard linear theory. Lotton et al. (2009) have presented an analytical modeling for predicting the transient temperature profile in thermoacoustic refrigerators. This modeling remains within the framework of the classical linear theory (Swift, 1988). In their modeling, the authors include the transverse heat conduction in the stack, the heat leakages through the duct walls, the heat generated by viscous losses in the stack, the heat generated by 
vorticity at the ends of the stack, and the heat transfer through both ends of the stack, along with the effects that are considered in the linear theory (Swift, 1988). The theoretical transient response of a thermoacoustic refrigerator is compared with experimental measurements. A good qualitative agreement is obtained between analytical and experimental results after fitting empirical coefficients. Poignand et al. (2006) have observed that optimal values of the temperature gradient, the thermoacoustic heat flow and the coefficient of performance of thermoacoustic refrigerators depend on the frequency, on the shape and the dimension of the stack, on the thermo-physical properties of the fluid and the stack plates, and on the acoustic pressure level in the resonator. A few of the studies consider the temperature field in the transient region and in different locations of the stack for different positions in the standing wave in order to understand the thermoacoustic effect. Experimental test data for high frequency thermoacoustic heat pumps are not sufficient, which is the motivation of the present study. Tasnim et al.(2010) have performed a systematic study on the influence of geometry, material, length and position of the stack on the performance of a thermoacoustic heat pump. The performance of the thermoacoustic heat pump, using stacks of different materials (Celcor ceramic, Reticulated Vitreous Carbon (RVC), and Mylar plastic), geometries (square cross section, open foam, and circular), lengths $(1 \mathrm{~cm}$ to 6.5 $\mathrm{cm}$, i.e., $0.01 \lambda$ to $0.068 \lambda)$, and positions $(0.5 \mathrm{~cm}$ to $8 \mathrm{~cm})$ is measured. The temperature measurements show that Celcor ceramic stack with square cross section gives the maximum performance regarding the temperature difference between the hot and cold end of the stack, and the hot end temperature with a stack length of $4 \mathrm{~cm}$ $(0.04 \lambda)$ and a stack center position of $4 \mathrm{~cm}$ from the pressure antinode (which is in agreement with the existing literature). But there exists different opinions about temperature distribution along a stack in different literatures. Reid and Swift (2000) have shown both experimentally and theoretically that temperature oscillations within a stack are linear for low temperature difference between the two ends of the stack. Their theoretical prediction is based on inviscid boundary-layer, short-stack approximations, and neglecting the ordinary conduction of heat down the temperature gradient, as it is small. The experimental measurement provided by the authors is only for a single stack location within a standing wave thermoacoustic refrigerator. Marx and Blanc-Benon (2005) have performed numerical investigations to calculate the temperature differences between the ends of a stack. The authors have compared the computed temperature difference to the one predicted by the linear theory at different Mach numbers, and at different positions of the stack from the pressure anti-node. The authors have observed some discrepancy even at low Mach numbers that are attributed due to thermal effects, and discrepancies at high Mach numbers are due to non-linear effects, especially temperature non-linearities that are generated in the vicinity of the stack. That is Marx and Blanc-Benon (2005) have observed non-harmonic temperature oscillations at a Mach number of $8 \%$ and at locations near the velocity anti-node. Gusev et al. (2001) analytically shows that the temperature oscillations near the edge of the thermoacoustic stack are highly anharmonic even in the case of harmonic oscillations in the thermoacoustic engines. The authors show that hydrodynamic heat flow along the stack generates thermal wave harmonics even in the absence of the higher harmonics of the acoustic field. The authors also emphasize that the existence of the high spatial temperature gradients should favor thermal wave harmonic generation. Therefore the present investigation targets to study the temperature fields inside and at the edges of stacks and the surrounding working fluid with an objective to improve the understanding of the physics underlying the thermoacoustic effect.

\section{EXPERIMENTAL APPARATUS}

Figure 2 shows a sketch of the thermoacoustic heat pump and the measuring systems. The description of the apparatus is also available in Tasnim et al. (2010). The device consists of a quarter-wavelength resonator having its fundamental resonance frequency at about 350 Hz. The resonator is built from a straight Pyrex tube of length $0.24 \mathrm{~m}$, internal diameter $2.1 \mathrm{~cm}$ and wall thickness $1 \mathrm{~mm}$. The working fluid is air at atmospheric pressure. One extremity of the tube constitutes the rigid end (reflector) of the resonator. At the other end a mid-range loudspeaker (Model NWX-516-8SQ) constituting the acoustic power source (driver). A function generator (GFG-8020H) and a power amplifier have been employed to drive the system at the operation frequency and with the selected power. Pressure measurements are made by a sound level meter (OMEGA HHSL1) very near the closed end in correspondence of a pressure antinode. Pressure measurements accuracy is estimable about two percent. A constant drive ratio $D=p_{1} / p_{m}=0.35 \%$ is used, where $p_{m}$ is the mean pressure and $p_{1}$ is the pressure amplitude at the pressure antinode. The selection of the

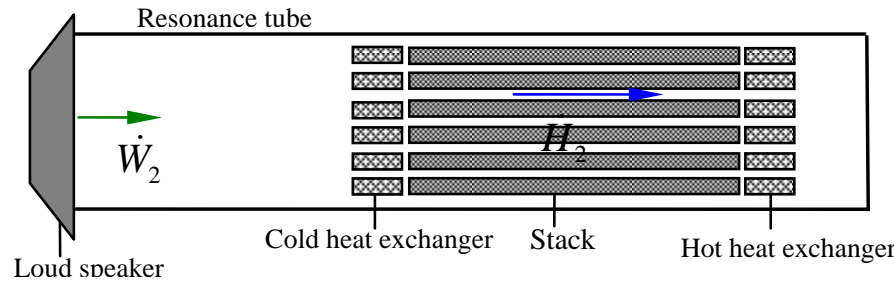

Fig. 1 A quarter wave length standing wave thermoacoustic heat pump with different components along with acoustic power $\left(\dot{W}_{2}\right)$ and energy flow $\left(\dot{H}_{2}\right)$ directions.

operating frequency of $350 \mathrm{~Hz}$, an average pressure of $100 \mathrm{kPa}$, and air as working gas determines the thermal and viscous penetration depths of $\delta_{k}=0.14 \mathrm{~mm}$ and $\delta_{v}=0.1 \mathrm{~mm}$, respectively. K-type chromega-Alomega thermocouples are used for temperature measurements with an accuracy of $\pm 0.1^{\circ} \mathrm{C}$, with a temperature range of ( 0 to $\left.482^{\circ} \mathrm{C}\right)$ and by using a data acquisition card (NIUSB-9211) via Lab VIEW software. For each experimental condition, three to four experimental runs are conducted for the consistency in the measured data. Taking into account for the reproducibility of the experimental measurements, the overall accuracy of the experimental data is of the order of ten percent.

The stacks used in the measurement set-up are the prefabricated stack made of 400-cpsi (cells per square inch) Corning Celcor ceramic material. Corning Celcor stack with a square cross section and four different lengths of $1 \mathrm{~cm}(0.01 \lambda), 2 \mathrm{~cm}(0.02 \lambda), 2.5$ $\mathrm{cm}(0.025 \lambda)$, and $4 \mathrm{~cm}(0.04 \lambda)$ are considered. In a recent study by Tasnim et al. (2010) reveals that regarding the maximum temperature at the hot side of the stack, and the maximum temperature difference between the hot and cold side of the stack, Corning Celcor stack of 4 $\mathrm{cm}$ length $(0.04 \lambda)$ shows the best performance. This is the reason for considering stack length up to $4 \mathrm{~cm}$ in the present study, since any extra surface area contributes to energy dissipation. Eight different positions (hot end of the stack, varying between $0.5 \mathrm{~cm}$ and $8 \mathrm{~cm}$ ) of the stacks from the pressure anti-node is measured. Tasnim et al. (2010) also shows that as the stack is moved away from the pressure anti-node, acoustic work absorbed by the stack plate increases, since velocity is increasing near the velocity anti-node, thereby increasing the viscous losses. Therefore, stack positions close to the pressure anti-node are focused in this study, and results are primarily presented near the pressure anti-node positions. The thermophysical properties of the stack are found in Tasnim et al. (2010).

\section{OPERATION PROCEDURE AND EXPERIMENTAL RESULTS}

The thermoacoustic effect along the stack is studied quantitatively recording as a function of time the temperature when the system is driven at its fundamental resonance frequency. This last has been 


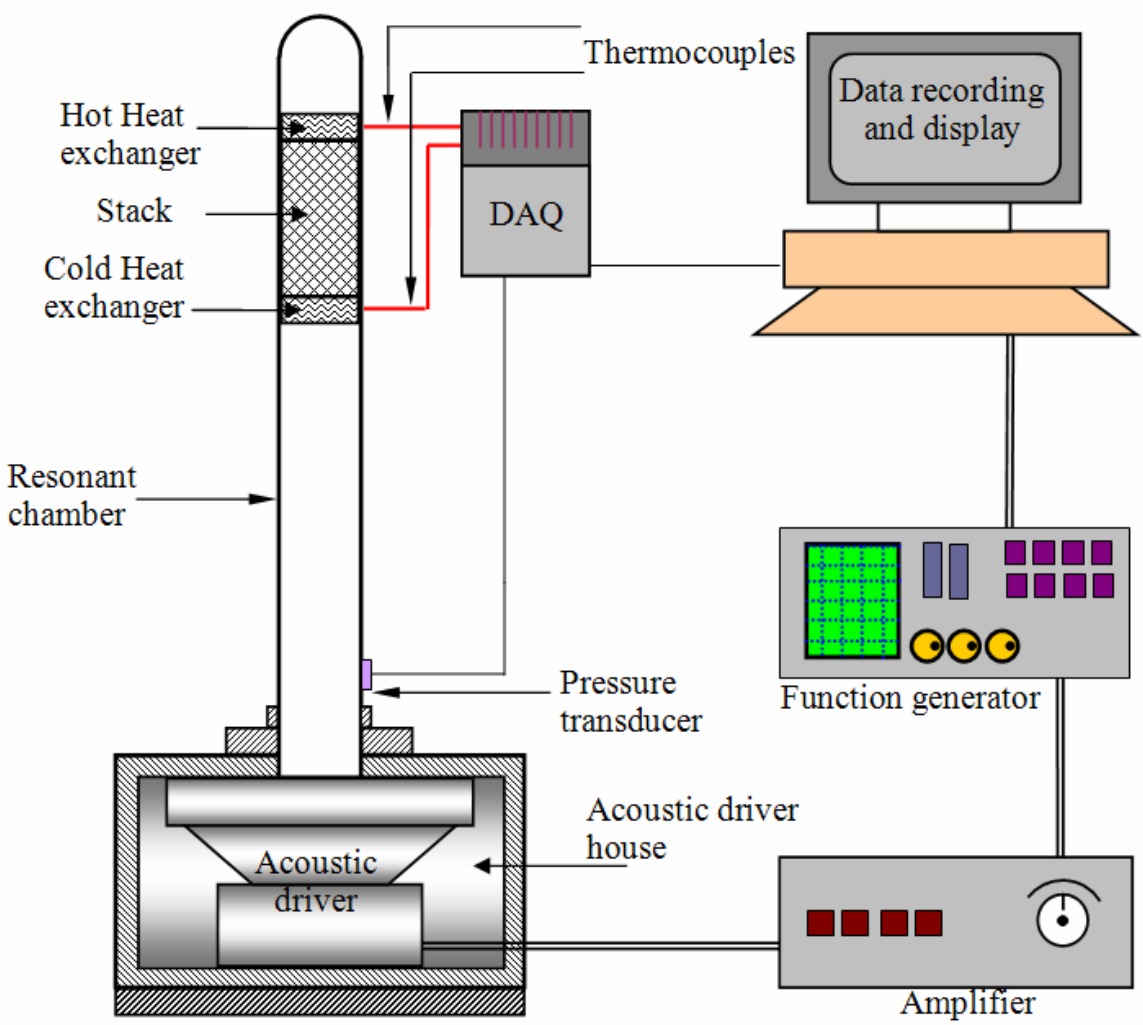

Fig. 2 Thermoacoustic heat pump and the measuring systems.

determined in a preliminary phase tuning the frequency at low dynamic pressure until the sound level meter signal reaches a maximum. At the beginning of each set of measurements the entire structure is at room temperature $(\sim 300 \mathrm{~K})$. Next, the thermoacoustic effect (hydrodynamic heat flow) is started when acoustic power is suddenly applied (at $t=0$ ). As no thermal reservoirs are connected to the stack ends, the thermoacoustic heat flow is sustained from the plates themselves. Each plate, therefore, will cool at $C$ (near the pressure node), where heat is removed and will heat at $H$ (near the pressure anti-node), where heat is reversed. Figure 3 shows typical experimental results of the time evolution of the temperatures measured at the extremities of the Corning Celcor stack which is $4 \mathrm{~cm}$ $(0.04 \lambda)$ long and the center of the stack is located $5 \mathrm{~cm}$ from the nearest pressure antinode. Right after the acoustic power is supplied, the temperature of the stack end that is close to the closed end of the tube starts to increase (as shown in Fig. 3) and the other end starts to decrease. After approximately half a minute, a temperature difference of $31^{\circ} \mathrm{C}$ is measured across the stack ends, with a cold side temperature of $13.8^{\circ} \mathrm{C}$ and a hot side temperature of $45^{\circ} \mathrm{C}$ as shown in Fig. 3. In this heat pump, the acoustic energy is supplied by the loudspeaker, and the gas particles near the stack plates (approximately a thermal penetration depth $\delta_{k}$ from the surface of the stack plates) absorb this energy. A hydrodynamic heat flow ( $\dot{Q}_{2}=\dot{H}_{2}-\dot{W}_{2}$ ) takes place in the gas near the surface of the stack plate and is directed from the stack end that is closest to the loudspeaker end to one that is near the pressure anti-node (as shown in Fig. 1). Because of this hydrodynamic heat flow, a temperature gradient develops across the stack with a cold end near the loudspeaker end and a hot end near the closed end. Figure 3 shows that the temperature difference across the stack increases rapidly in the early stages (during the first 10 seconds) of the hydrodynamic heat flow process, where the growth is highest. In this early stage of hydrodynamic heat flow process, the diffusive heat flux from the hot end of the stack to that of cold end is not significant. After approximately 30 seconds, the temperature curves become flat, clearly reflects the increasingly role played by a diffusive heat flux through the stack plates and the gas from hot to cold end which tends to balance the hydrodynamic one. The diffusive heat flow driven by the temperature difference is zero at the initial time where the temperature curves exhibit their maximum slope and maximum at the

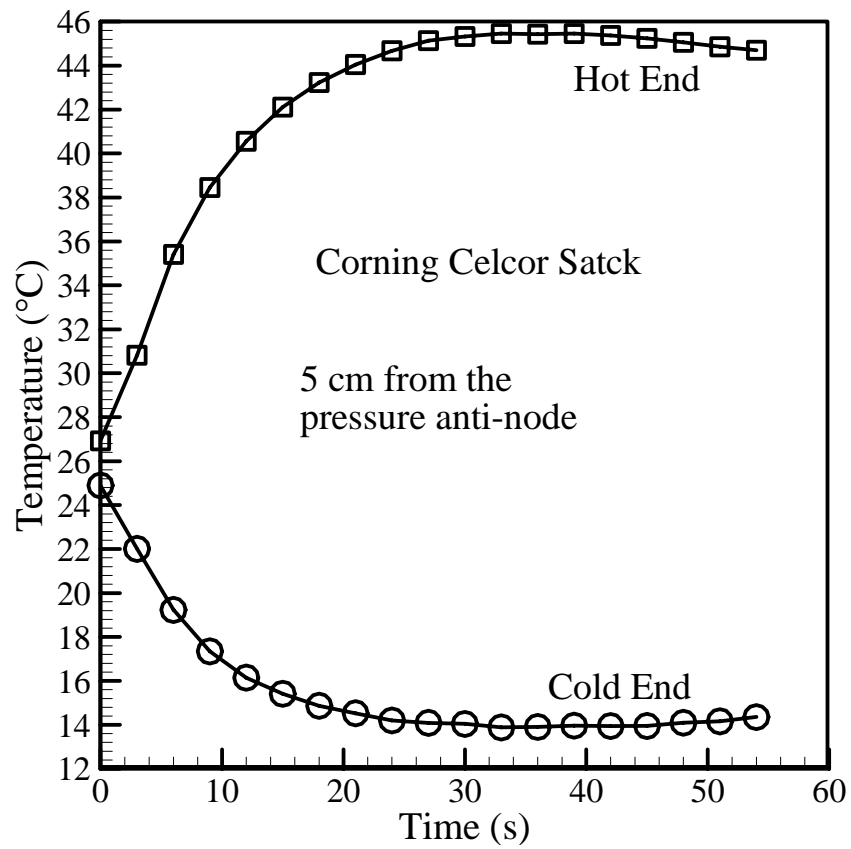

Fig. 3 Time evolution of the measured temperatures at different locations on the stack when the $4 \mathrm{~cm}$ Celcor stack is placed 5 $\mathrm{cm}$ from the nearest pressure antinode. 
steady state when curves become flat. The temperature slopes are linear right after the device has started; within the first five seconds. During the first few seconds, temperature is almost uniform through the entire system; therefore, there are no limiting mechanisms for the heat flow. After that, the linear behavior disappears as temperature difference starts to develop along the stack, and heat flow is starting to be balanced by the limiting mechanisms. The hot and cold end temperature profiles are not symmetric with respect to the center of the stack. Since acoustic power is absorbed by the gas near the stack to transport heat from the low temperature side to that of the hot one, this absorbed acoustic energy shows up as dissipative heat (through viscous and thermal processes) at the hot side of the stack. Therefore, a higher temperature increase at the hot side of the stack is observed.

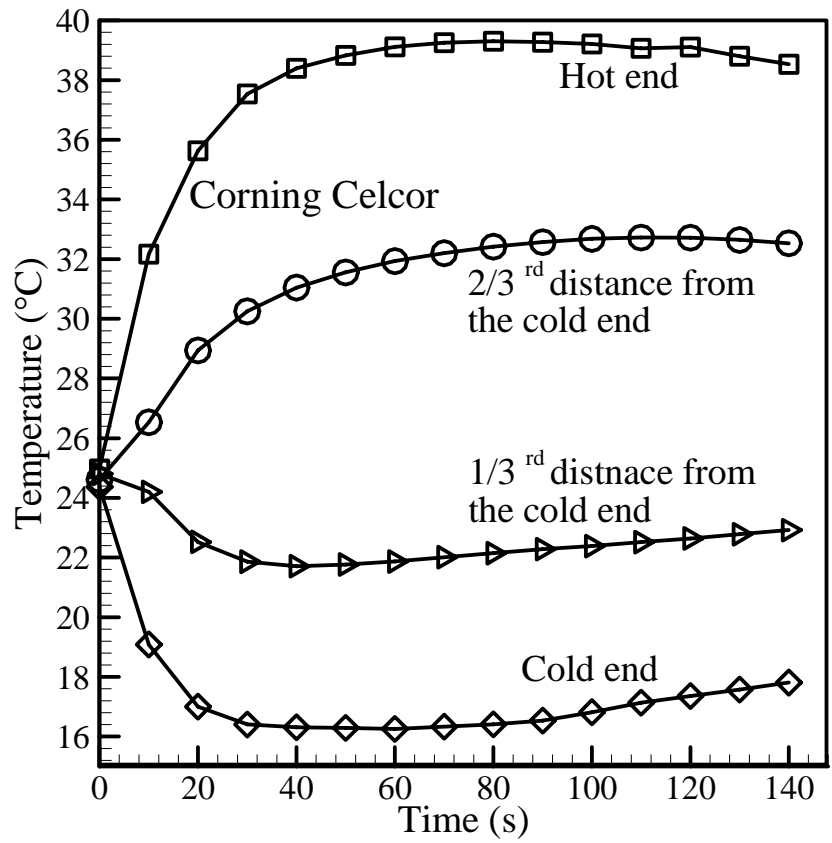

Fig. 4 Time evolution of the measured temperatures at different locations on the stack when the $4 \mathrm{~cm}$ stack is placed $3 \mathrm{~cm}$ from the nearest pressure antinode.

Figure 4 presents the time evolution of the temperatures measured on four different locations of the Corning Celcor stack which is $4 \mathrm{~cm}$ long and the center of the stack is located $4 \mathrm{~cm}$ from the nearest pressure antinode. The slopes of temperatures at different locations on the stack except at the ends indicate less heat transfer between the fluid and the plate compared to the two extreme ends (in Fig. 4). The heat pumped by the cold end of the stack to the hot end is balanced by the return conductive heat transfer in an isolated stack, therefore, a less conductive heat transfer in any region of the stack indicates a high convective heat transfer between the stack and the working fluid in that location. Thus high convective heat transfer is observed between the stack and the working fluid near the ends of the stack compared to the other locations.

Figure 5 (a) to (d) show the time evolution of the temperatures measured at four different locations on the Corning Celcor stack that are $1 \mathrm{~cm}(0.01 \lambda), 2 \mathrm{~cm}(0.02 \lambda), 2.5 \mathrm{~cm}(0.025 \lambda)$, and $4 \mathrm{~cm}(0.04 \lambda)$ long respectively, and the center of the stacks are located 1.5, 2, 2.5, and $3 \mathrm{~cm}$ from the nearest pressure antinode. That is for all of the above mentioned stack lengths, the hot end of the stack is located $1 \mathrm{~cm}$ from the pressure anti-node. Temperature measurements at other positions of the stack from the pressure anti-node show the similar profiles for the respective stack lengths. The slopes of temperatures at different locations on the stack except at the ends indicate less heat transfer between the fluid and the plate compared to the two extreme ends (in Figs. 5(a) to (d)). The temperature measurements with time for $1 \mathrm{~cm}$,
$2 \mathrm{~cm}$, and $2.5 \mathrm{~cm}$ stack lengths show the similar profiles, i.e., less conductive heat transfer is observed between $1 / 3^{\text {rd }}$ and $2 / 3^{\text {rd }}$ locations of the stack from the cold end, and a high conductive heat transfer is observed between the hot end and the $2 / 3^{\text {rd }}$ location of the stack from the cold end. Except for the $4 \mathrm{~cm}$ long stack, a high temperature difference is observed between $1 / 3^{\text {rd }}$ and $2 / 3^{\text {rd }}$ length of the stack from the cold end location. Figures 5(a) to (d) show that time evolutions of the measured temperatures at different locations on the stack changes as the length of the stack changes. Also, the steady state temperature difference produced at the stack extremities is different for different lengths (observed in a previous article by Tasnim et al. (2010)).

Temperatures along the $2.5 \mathrm{~cm}$ long stack at four different locations indicate almost linear profile along the stack in Fig. 6 (a). Figure 6 (a) shows the temperature distribution along the stack plate at three positions of the stack from the pressure anti-node. The dashed lines are linear fit through the data points. Gusev et al. (2001) in their analytical investigation indicated a non-linear behavior of temperature along the axial position near the extreme ends. This variation of temperature at the ends is not observed in the present experimental conditions. During this measurement the stack hot end is moved from $0.5 \mathrm{~cm}$ to $8 \mathrm{~cm}$ from the nearest pressure anti-node (to organize, all the positions of the stack are not shown in Fig. 6 (a)). Figure 6(a) shows that near the pressure anti-node, temperature along the stack is almost linear (stack position 1and $2 \mathrm{~cm}$ in the figure). But, as the stack is moved away from the pressure anti-node (stack position $6 \mathrm{~cm}$ in the figure); the temperature along the stack becomes linear. For the two furthest positions of the stack from the pressure anti-node $(6 \mathrm{~cm}$ and $8 \mathrm{~cm})$, the temperature profiles show linear behavior through out the stack. This information is not reported in the earlier literature. An important information reveals from this figure is that as the stack is moved from the pressure anti-node (after $5 \mathrm{~cm}$ ), temperature slope along the stack becomes flatter showing less temperature difference along the stack. Figure 6 (b) for $2 \mathrm{~cm}$ long stack follows the similar trend as observed in Fig. 6(a). Figure 6 (c) for $1 \mathrm{~cm}$ long stack at a position near the velocity anti-node $(6 \mathrm{~cm})$ does not present any improvement of linear behavior of temperature which is observed earlier in Fig. 6 (a) and (b). Therefore, the improvement of linear behavior near the velocity anti-node is not applicable for all the stack lengths considered.

Figure 7 presents the time evolution of the stack and the corresponding gas parcels temperatures at four different locations on the stack. The gas temperatures are measured at a distance of 0.2 $\mathrm{mm}$ from the stack at each location. Note that for the measured operating frequency, pressure, and fluid properties, the thermal penetration depth calculated for the present study is $0.14 \mathrm{~mm}$. Location" 1 " indicates cold end of the stack, where the corresponding gas temperature is lower than the stack temperature, indicating heat transfer from the cold stack end to the gas. A similar heat transfer phenomenon occurs at location " 2 ". Location " 4 " is the hot end of the gas, where gas temperature is higher than that of the stack, indicating heat transfer from the hot gas to the stack. Similarly at location "3", heat transfer occurs from the hot gas to the stack. This result shows the thermoacoustic heat transfer phenomena that are not available in the existing literature. The corresponding steady state temperatures are shown in Fig. 8 (a), where heat flow direction is reversed for the two halves of the stack. The error bars are showing uncertainties in the thermocouple readings. Figure 8 (a) is very insightful as it provides steady state heat transfer information between the stack and the nearby gas both at the edges and at other locations. This information is not available in the existing literature. A higher temperature difference between the stack and the corresponding gas indicates a higher convective heat transfer at the hot end compared to the cold end of the stack. This information is in agreement with the high temperature increase at the hot end of the stack in Figs. 3 to 6 and also the discussions provided in Section 3. A high temperature difference between the stack and the gas at the edges disappears at 


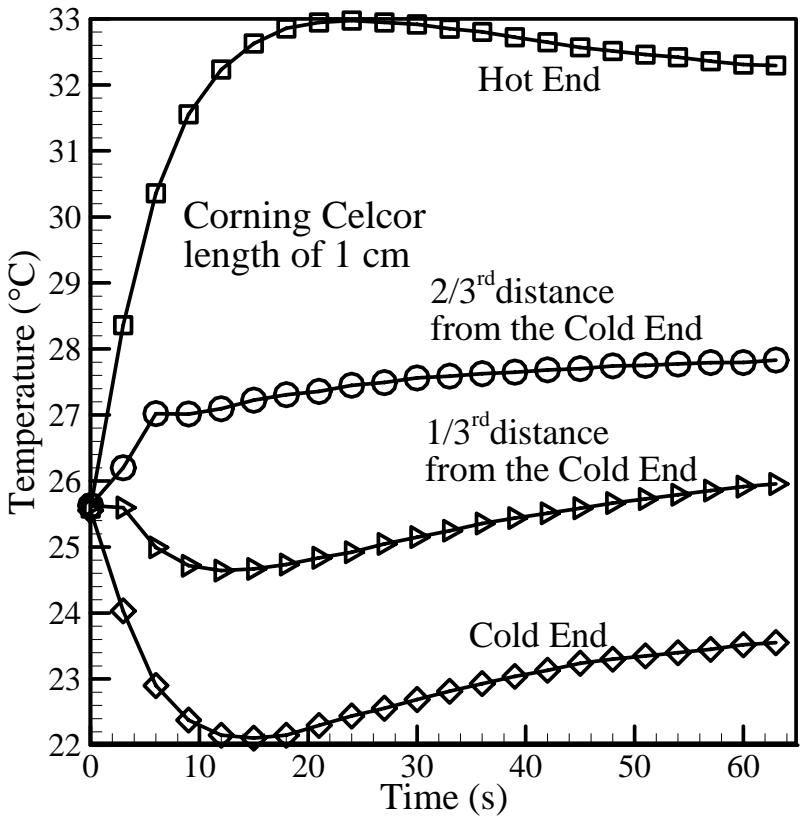

(a)

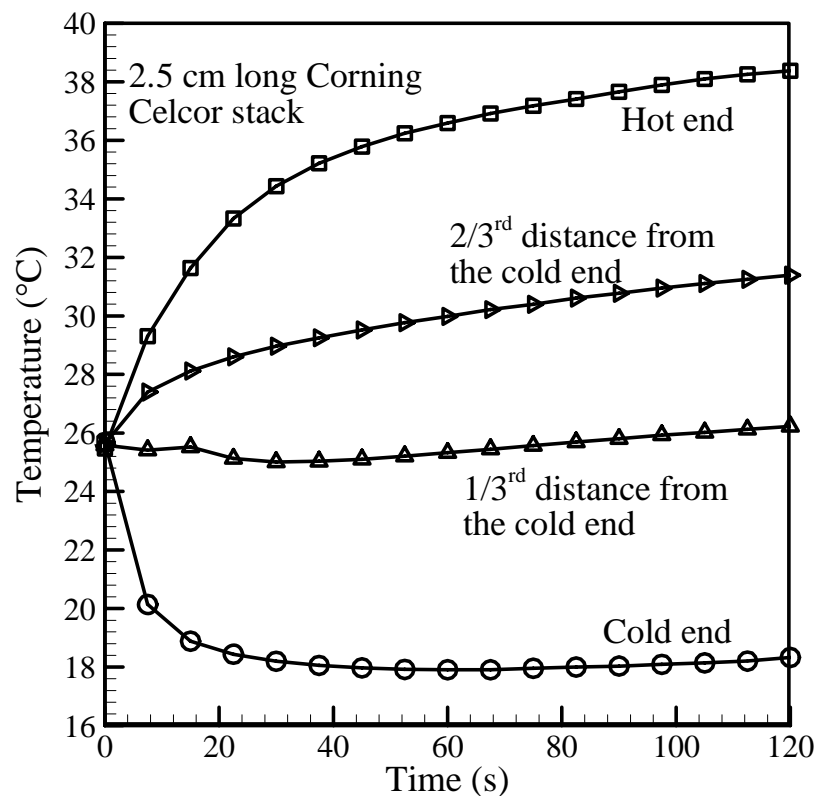

(c)

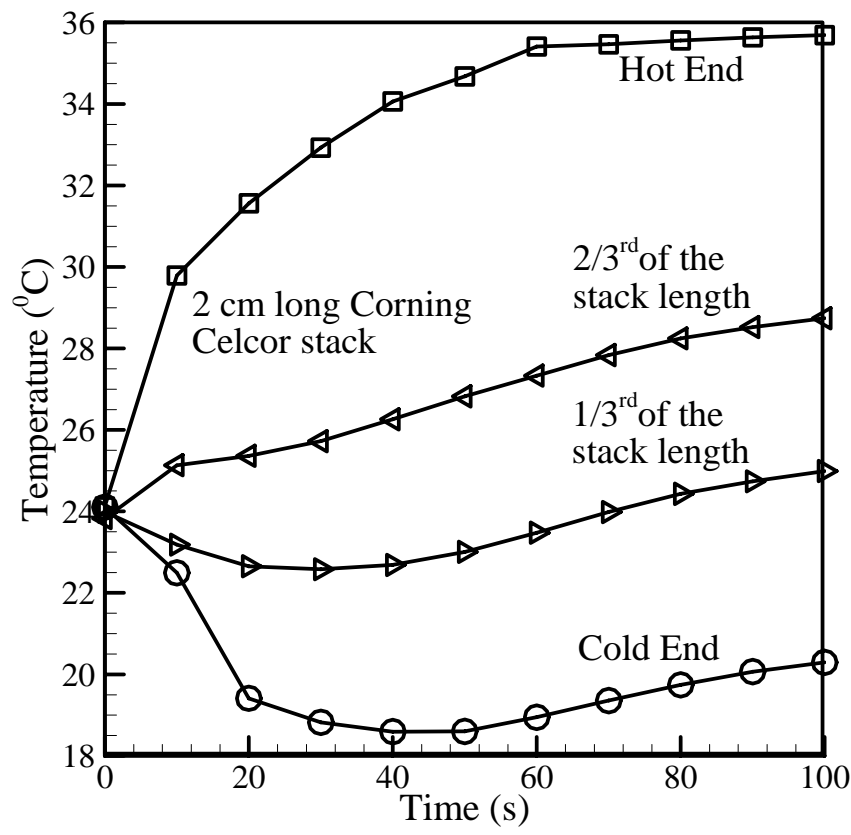

(b)

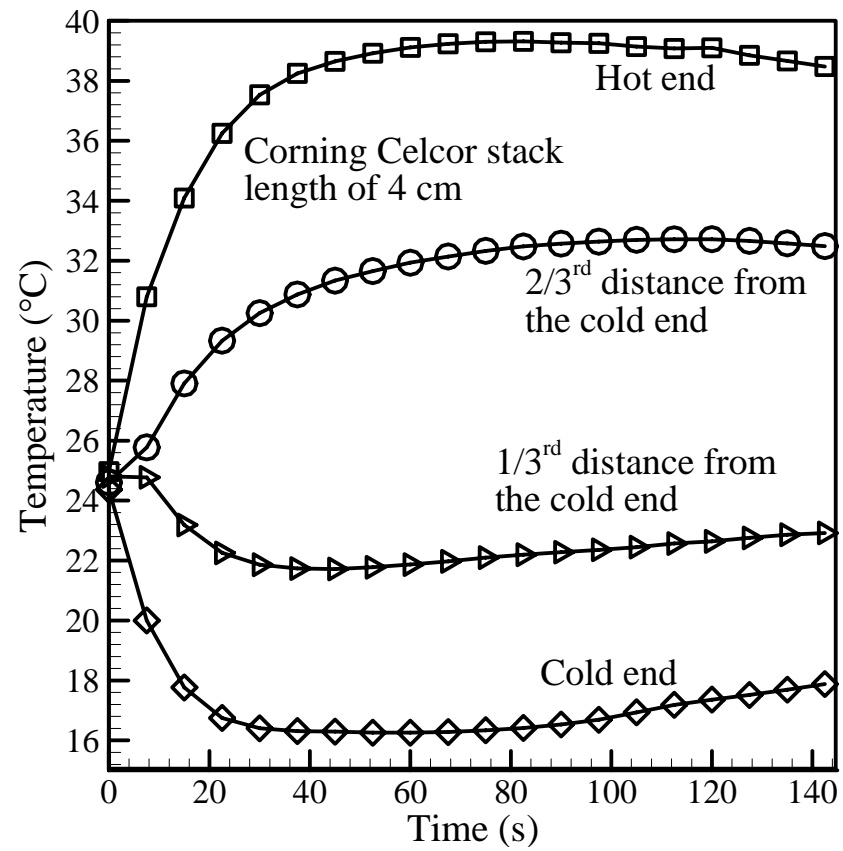

(d)

Fig. 5 Time evolution of measured temperatures at different locations on the stack when (a) $1 \mathrm{~cm}$ stack is placed $1.5 \mathrm{~cm}$ and (b) $2 \mathrm{~cm}$ stack is placed $2 \mathrm{~cm}$ from the nearest pressure antinode(c) $2.5 \mathrm{~cm}$ stack is placed $2.5 \mathrm{~cm}$ and (d) $4 \mathrm{~cm}$ stack is placed $4 \mathrm{~cm}$ from the nearest pressure antinode.

other locations on the stack. This high temperature difference between the stack and the gas indicates high convective heat transfer at the edges that gradually decreases as one moves away from the edge, becomes zero right at the middle of the stack, and then changes its direction for the other half of the stack. Therefore, there is non-uniformity of the thermoacoustic process along the stack. Figure 8(a) supports Fig.11 (a) of Swift (1988) that describes the gas parcel model of thermoacoustics. The right part of the temperature profile from the center of the stack in Fig. 8(a) corresponds to the second step, and the left part of Fig. 8 (a) corresponds to the fourth step of Fig. 11(a) of Swift (1988). A higher temperature slope is observed for the gas parcels than the stack, and this phenomenon is due to thermoacoustic effect. Therefore, the prediction of linear thermoacoustic theory for calculating enthalpy flow along the stack or evaluating the temperature difference across the stack using similar temperature gradient along the stack plate and the nearby working gas is not perfect. A higher temperature gradient along the gas should be considered than along the stack. This information is not available in the existing literature. The temperatures along the stack indicate a higher horizontal temperature difference between the edges and the nearby locations and lower temperature difference between the other two locations. This information is in agreement with Fig. 5(b) and (c) that present the time evolution of the measured temperatures at different locations on the stack when the 2 and 2.5 $\mathrm{cm}$ stacks are placed $1 \mathrm{~cm}$ from the nearest pressure antinode. The lines through the data points are linear fit that indicate linear temperature profile along the gas and the stack. In Figs. 7 and 8(a), the center of the $2 \mathrm{~cm}$ stack is located $3 \mathrm{~cm}$ from the nearest pressure 


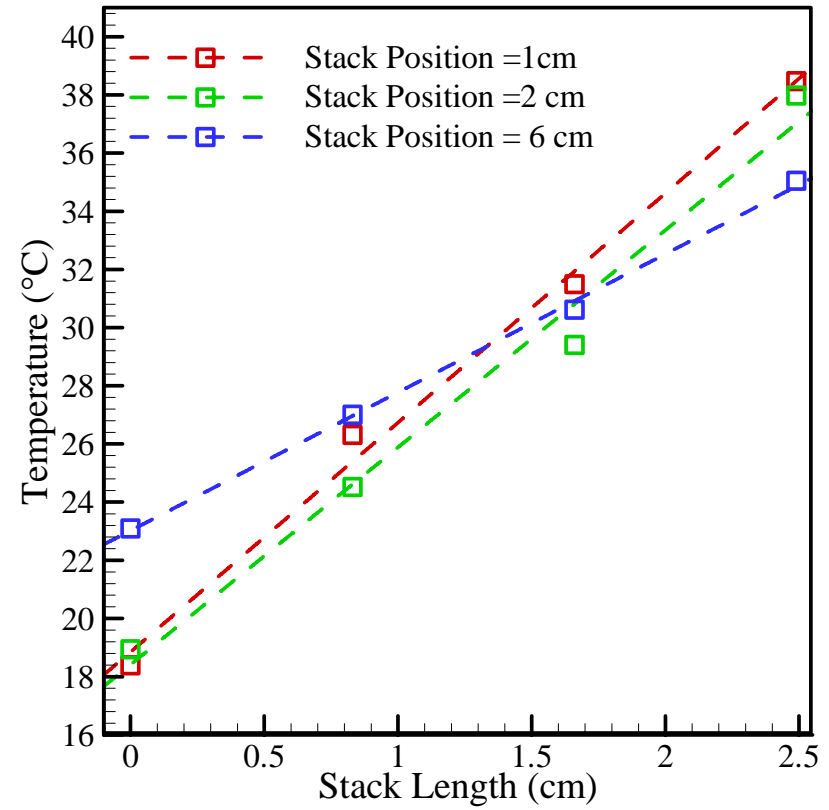

(a)

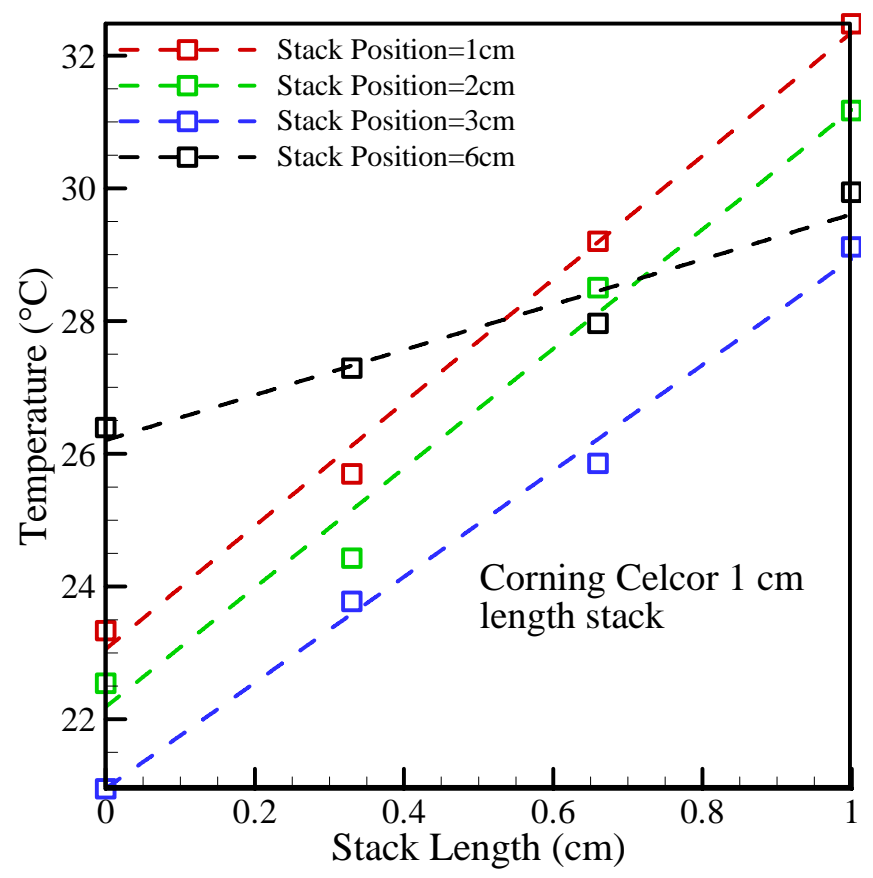

(c)

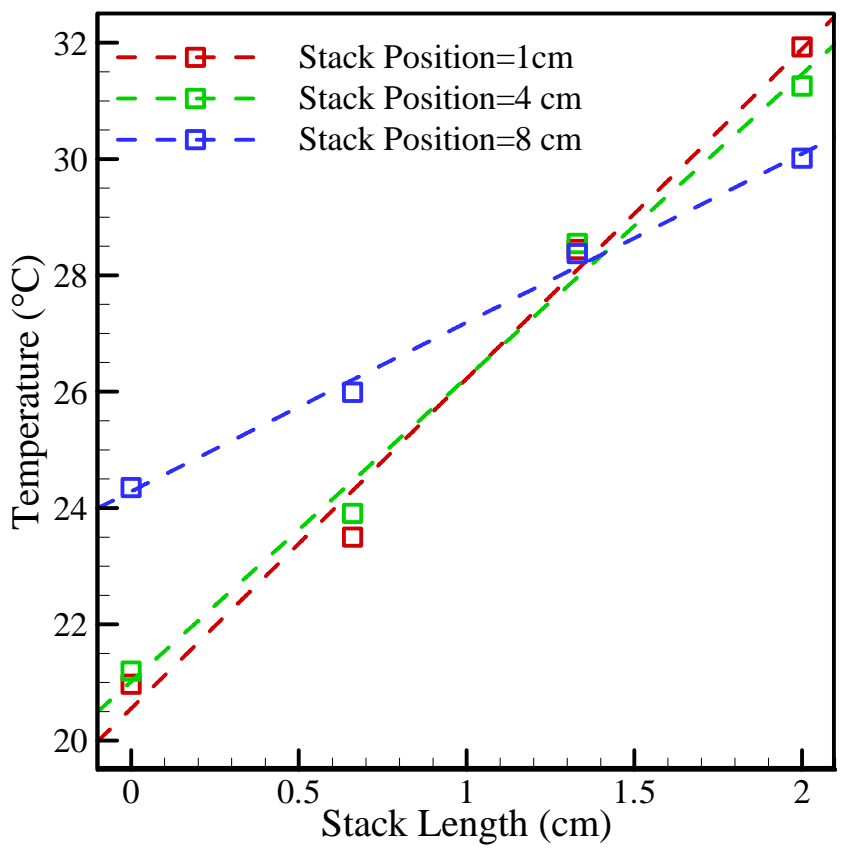

(b)

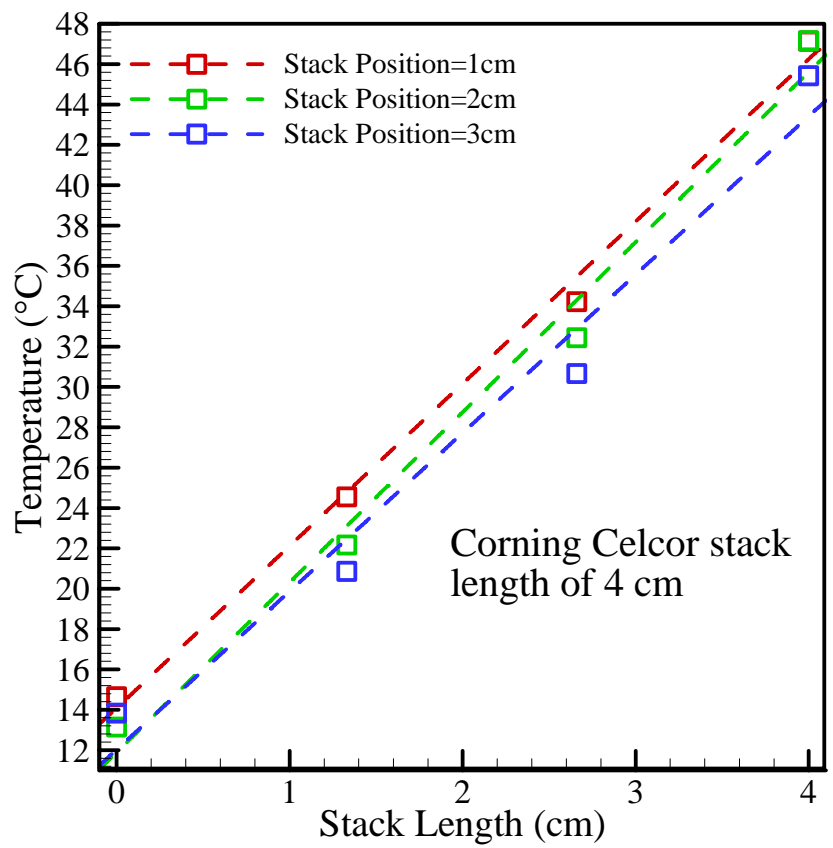

(d)

Fig. 6 Temperature along the stack length; (a) stack length 0 indicates starting of the stack and $2.5 \mathrm{~cm}$ the end of the stack. " 0 " indicates the cold end and " 2.5 " the hot end of the stack, and (b) stack length 0 indicates starting of the stack and $2 \mathrm{~cm}$ the end of the stack; (c) stack length 0 indicates starting of the stack and $1 \mathrm{~cm}$ the end of the stack.” 0 ” indicates the cold end and " 1 ” the hot end of the stack, and (d) stack length 0 indicates starting of the stack and $4 \mathrm{~cm}$ the end of the stack. The dashed lines are the linear fit through the data points.

anti-node. Figure 8(b) shows the time averaged transverse heat flow due to the thermoacoustic effect along the stack based on the temperature measurements in Fig. 7 and 8(a). The heat flow between the surface of the stack and the oscillating fluid is maximum at the edges, changes its direction at the middle of the stack plate due to the temperature distributions along the stack plate surface and the oscillating fluid. Because of this transverse heat flow, energy flow in the oscillating flow (longitudinal) direction is impossible to keep constant (the assumption of constant energy flow in the longitudinal direction is assumed in the linear theory Swift (1988)). Figures 9 (a) and (b) show the steady state temperature distributions along the 2 $\mathrm{cm}$ long stack and the nearby fluid when the stack center is located 4 $\mathrm{cm}$ and $5 \mathrm{~cm}$ from the nearest pressure anti-node. The temperature gradient along the fluid is higher than the stack for all the selected positions. The difference in temperature gradient developed along stack and the fluid is not affected by the location of the stack center from the pressure anti-node.

Figure 10 (a) shows the time evolution of temperatures at two extreme ends of the stack and the corresponding gas that are 0.4 $\mathrm{mm}$ from both ends. The particle displacement amplitude at the stack center end is $0.4 \mathrm{~mm}$. Figure 10 (a) is very perceptive, location " 2 " is the cold and " 3 " is the hot end of the stack. Near the cold end gas 


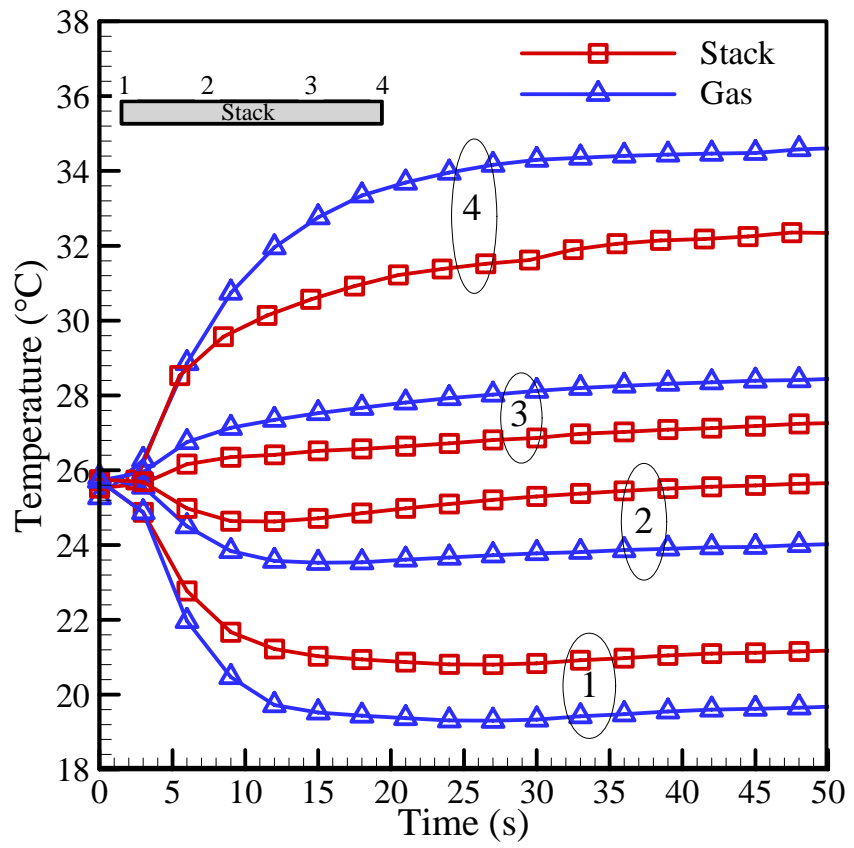

Fig. 7 Time evolution of temperature measurements at four different locations in the $2 \mathrm{~cm}$ stack and in the gas. " 1 " and " 4 " indicate cold and hot side of the stack, " 2 " and " 3 " are $0.67 \mathrm{~cm}$ and $1.33 \mathrm{~cm}$ from the cold end, respectively.

temperature is higher than the stack end temperature. Therefore, heat will flow from the cold gas to the cold stack end. On the other hand, near the hot stack end, gas temperature is lower than the stack end temperature. Therefore, heat will flow from the hot stack end to the gas. The temperature measurements at the stack extremities and the neighboring gas show axial heat transfer at the stack extremities. This information is a clear challenge to the hypothesis of a perfectly isolated stack used in the linear thermoacoustic theory. According to linear thermoacoustic theory, the stack edges are perfectly isolated, i.e., no heat is allowed to enter or leave either axially or transversally through the ends. This figure also supports the thermoacoustic heat pump process between the gas that resides near the edges of the stack and the heat exchangers. A schematic diagram illustrating the temperature distribution in a thermoacoustic heat pump is presented in Fig. 10 (b). The arrows indicate thermoacoustic heat flow between the elements. When designing a thermoacoustic device, the major theory to rely upon is the linear theory proposed by Swift (1988). The linear theory (Swift (1988)) for thermoacoustic devices considers energy flow between the stack and the heat exchangers, but not the mechanism for heat flow. Since the heat transfer between the stack and the heat exchangers depends on the finite displacement of the working fluid and therefore is beyond the scope of the linear theory treatments. Four mechanisms have been identified in the present study by which heat can transfer between the stack ends and the nearby heat exchangers. They are as follows:

1. conduction heat transfer (as shown in Fig. 10 (a), since there is temperature variation between the stack extremities and the nearby gas that are at a position of particle displacement amplitude from the stack ends. According to Marx and Blanc-Benon (2004), to obtain maximum cooling load for a thermoacoustic refrigerator, a heat exchanger should be placed at a distance of particle displacement amplitude from the stack ends.)

2. heat transfer from the stack ends to the heat exchangers with a heat transfer coefficient (h, as shown in Fig. 10 (a)). Estimation of " $h$ " between the stack and the walls of thermoacoustic heat exchangers is considered an unsolved problem and a major challenge in the design of heat exchangers. Several authors Garrett et al. (1994), Mozurkewich (2001), Swift (2002)) have suggested different approaches to estimate "h". According to Lotton et al. (2009), the value of "h" depends on the position of the stack in the resonator. The value of " $h$ " is increased when the stack is moved away from the pressure anti-node, near the center of the resonator. Since the velocity amplitude is increasing near the velocity anti-node. Brewster et al. (1997) have proposed an expression for calculating the heat transfer coefficient between the stack ends and the heat exchangers. According to Brewster et al. (1997), the value of "h" is proportional to the amplitude of the acoustic wave. Convection is the dominant mechanism of heat exchange between elements of a thermoacoustic device that is forced by the action of the acoustic oscillation.

3. thermoacoustic heat flux (as shown in Fig. 11)

4. heat transfer due to non-linear phenomenon at the ends of the stack (as shown in Fig. 12).

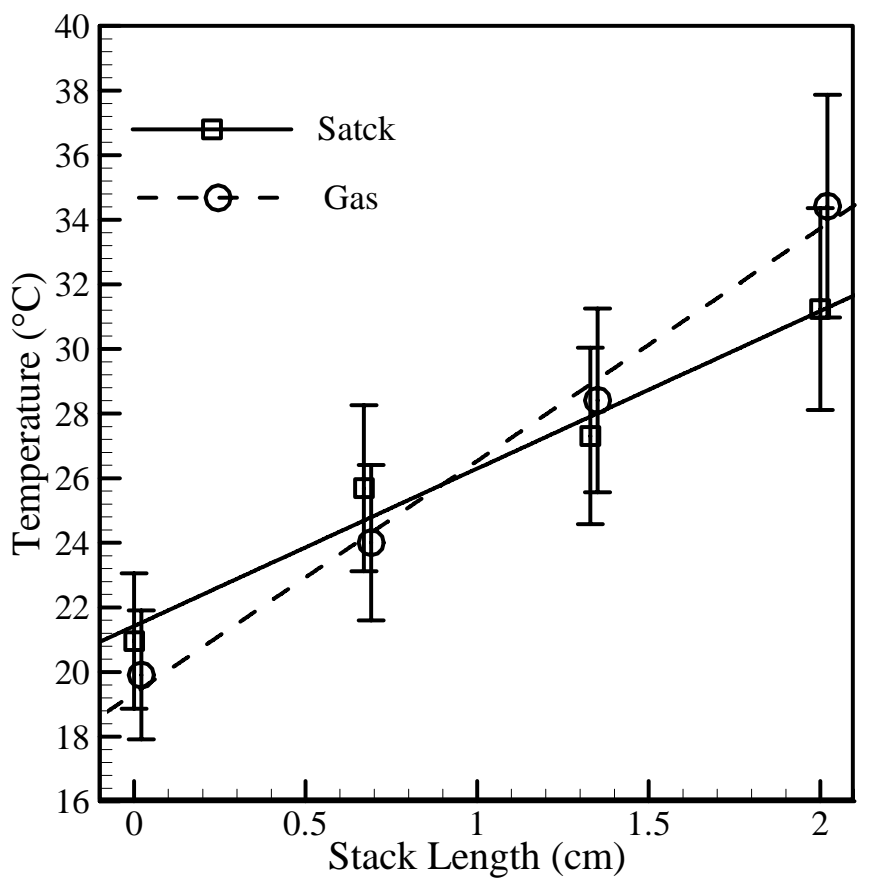

(a)

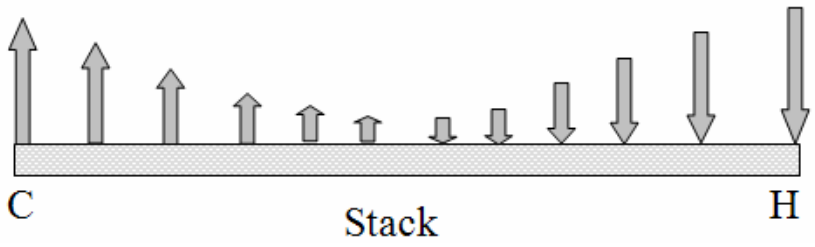

(b)

Fig. 8 (a) Steady state temperature along the stack and the gas; stack length $0 \mathrm{~cm}$ indicates starting of the stack and $2 \mathrm{~cm}$ the end of the stack." 0 " indicates the cold end and " 2 " the hot end of the stack. The solid and dashed lines are linear fit through the data points, and (b) schematic diagram of the directions of the vertical heat flux along the stack based on the measurements. "C" and " $\mathrm{H}$ " are cold and hot sides of the stack, respectively.

Let's consider a heat exchanger at a distance of particle displacement amplitude right next to the hot end of the stack, so this is the hot exchanger that is positioned close to the pressure anti-node. Heat exchangers are required in thermoacoustic devices to supply and extract heat at the ends of the stack. These heat exchangers are kept isothermal by flowing heat exchange fluids, heat pipes, or by using 


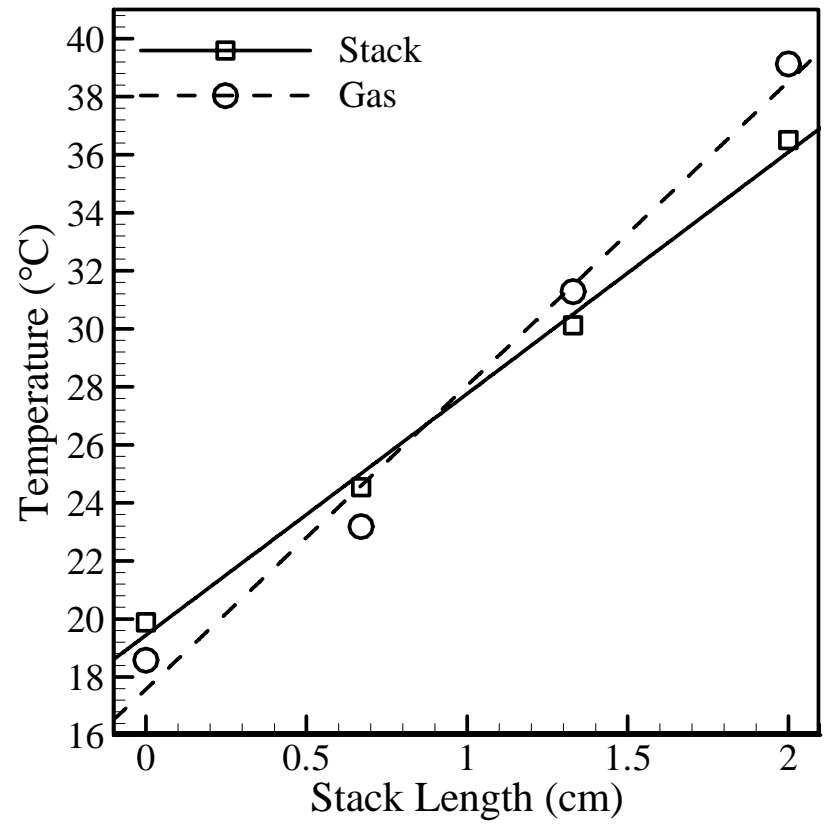

(a)

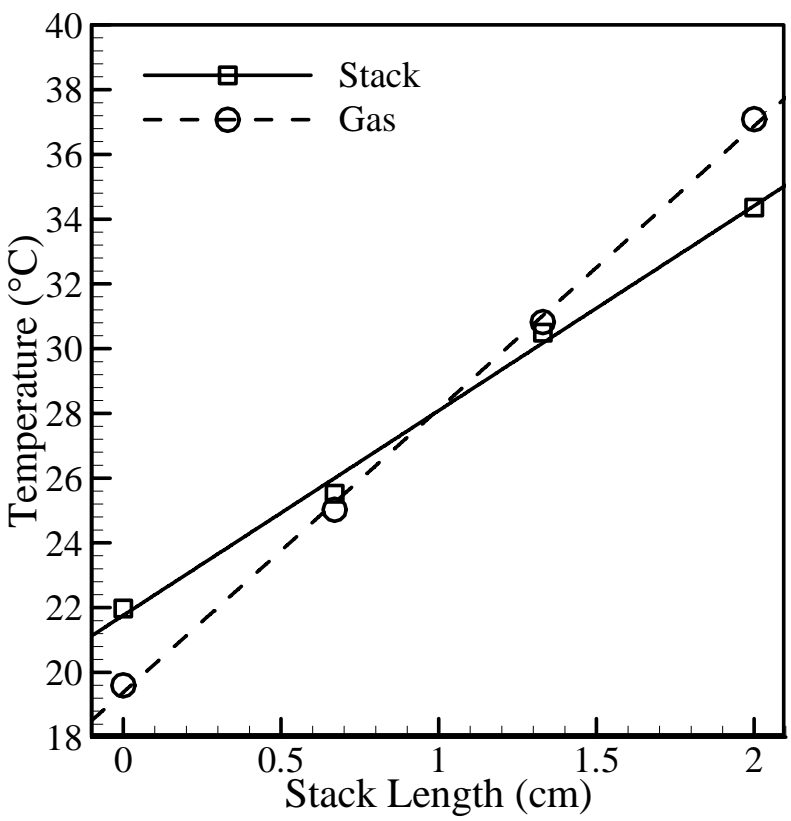

(b)

Fig. 9 Steady state temperature along the stack and the gas; stack length $0 \mathrm{~cm}$ indicates starting of the stack and $2 \mathrm{~cm}$ the end of the stack. " 0 " indicates the cold end and " 2 " the hot end of the stack. The solid and dashed lines are linear fit through the data points. (a) the stack center is 3 $\mathrm{cm}$ and (b) $4 \mathrm{~cm}$ from the nearest pressure anti-node.

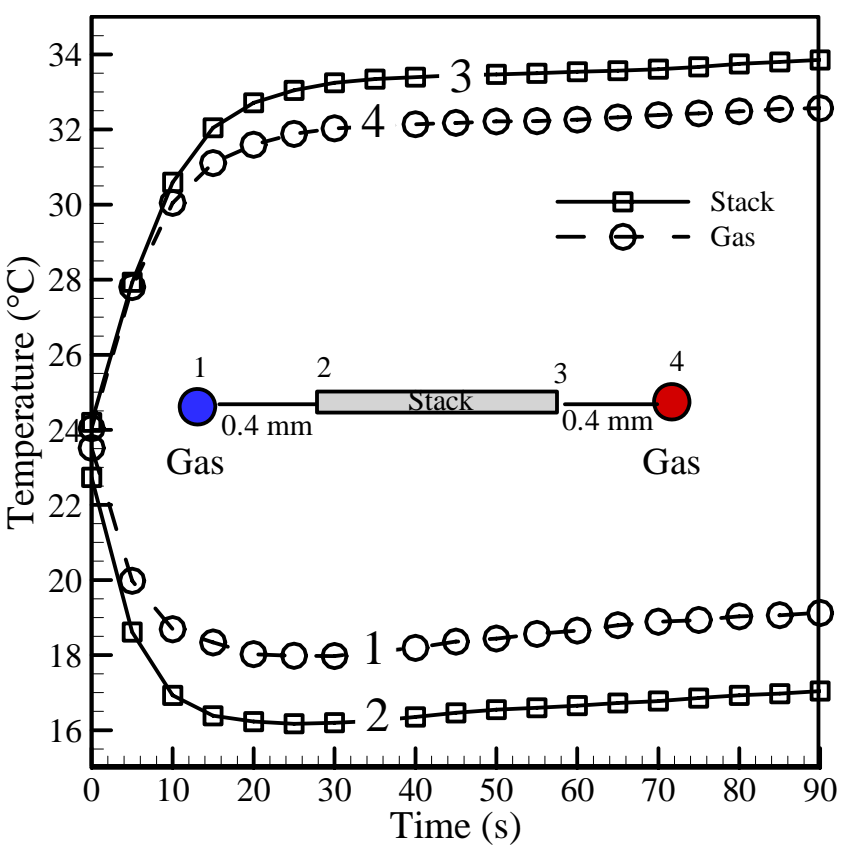

(a)
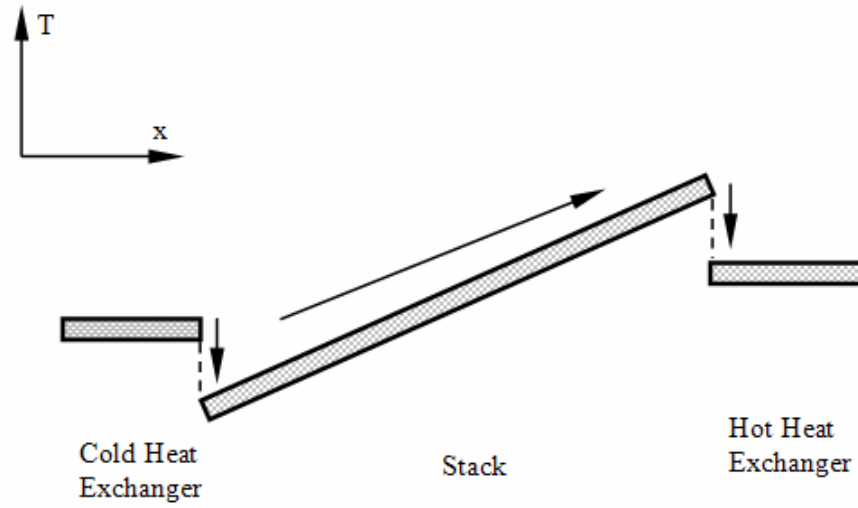

(b)

Fig. 10 (a) Time evolution of temperature measurements at extremities of $2 \mathrm{~cm}$ long stack. Gas temperatures are measured at $0.4 \mathrm{~mm}$ from both stack ends. " 2 " is the cold and " 3 " is the hot end of the stack. Stack center is placed $4 \mathrm{~cm}$ from the pressure anti-node, and (b) a schematic diagram illustrating the temperature distribution in a thermoacoustic heat pump. The arrows indicate thermoacoustic heat flow between the elements.

perfect heat conductors. In Fig. 11 , " $x_{1}$ " is the gas particle distance amplitude, which is the distance a fluid/gas can oscillate during the half acoustic cycle, so $2 x_{1}$ is the total distance a gas travels during an acoustic cycle. $u_{1}$ is the fluid oscillation amplitude at the velocity anti-node and $\omega$ is the angular frequency of oscillation. $T_{H}$ is stack hot end temperature and $\delta_{k}$ (thermal penetration depth) is the length perpendicular to the direction of oscillations of the gas. At a distance much larger than the thermal penetration depth from the solid boundary, the gas feels no thermal contact with the solid boundaries.
Let's consider a magnified view of a gas particle at the stack edge as it completes an acoustic cycle. According to Fig. 10 (a), the hot heat exchanger temperature in Fig. 11 is lower than the stack hot end temperature. The temperature of the gas particle is higher than the stack end temperature at that location (as shown in Fig. 8 (a)). At the rightmost position (state 2) of the parcel of gas shown in Fig. 11 during the first half of the cycle, it rejects heat to the heat exchanger, because its temperature is raised above the local heat exchanger temperature by isothermal compression. Stage 2 to 3 is the thermal equilibrium period, at this period the gas parcel temperature becomes 


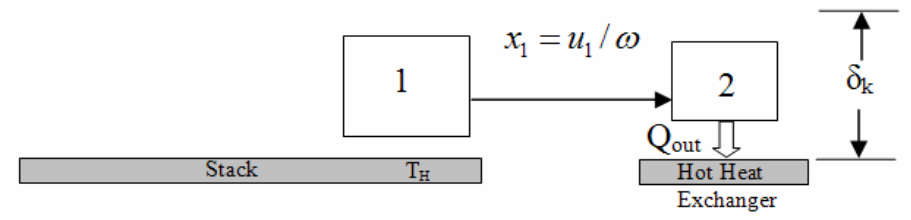

First half of a cycle

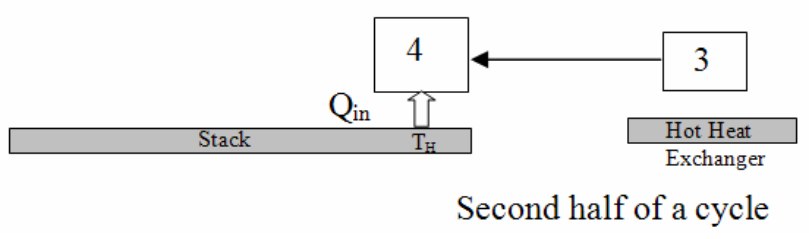

Fig. 11 Magnified view of a gas parcel as it completes an acoustic cycle.

equal to the heat exchanger temperature. At the leftmost position (state 4) during the second half of the cycle, it extracts heat from the stack hot end, as the gas parcel's temperature is less than the stack hot end temperature due to isothermal expansion. So, a gas parcel near the stack edge delivers some heat to the hot heat exchanger during the first half of the acoustic cycle and accepts some heat from the stack edge during the second half of the acoustic cycle. Therefore, some heat is moved from the stack hot end to the nearby hot heat exchanger due to thermoacoustic effect. Thus a thermoacoustic heat pumping effect is observed between the gas that resides near the edges of the stack and the heat exchangers.

The $x$-component of time averaged velocity $\left(u_{1}\right)$ (averaged over few cycles) is presented in Fig. 12 at different locations on the stack. This is a numerical result that is obtained after solving the compressible Navier-Stokes, continuity, and energy equations for an ideal gas (see reference (2009) for details numerical simulations, geometric, operating and boundary conditions). The objective of the numerical simulations is to calculate the heat and energy flows through thermoacoustic couple (a parallel-plate stack without heat exchangers much shorter than the acoustic wavelength), for which flow and thermal fields' information are required. A simple case is treated in this case for the numerical simulations, with zero longitudinal temperature gradient along the stack plate (as if the plates are made of a solid with infinite thermal conductivity and heat capacity). Figure 12 is part of those numerical simulations results that presents time averaged fluctuating velocity along the transverse direction at the left and right end locations on the stack. Vertical distance zero indicates the surface of the plate, where velocity fluctuation is zero at all locations because of the no-slip boundary condition, and the maximum vertical location indicates half gap $\left(y_{0}\right)$ between two stack plates. Large circulations are observed at the ends of the stack, changing the magnitude of fluctuating velocity from positive to negative at the left end and negative to positive at the right end. That means the circulations are at the opposite directions at the two ends. The reason for these circulations is due to the abrupt change of boundary condition at the stack edges from no-slip to slip. This figure shows that heat can transfer due to non-linear phenomenon (such as circulations/vortices) at the ends of the stack. This vortex formation near the edges could generate transverse heat transfer rates greater than the ones that would be obtained uniquely by thermal conduction. Optimal design of heat exchangers in thermoacoustic devices depends on the understanding of these thermo-fluid processes controlling the critical heat transfer between the sound wave and the heat exchangers at the stack-heat exchanger interfaces.

The estimated COPR (coefficient of performance of a thermoacoustic heat pump (estimated according to the linear

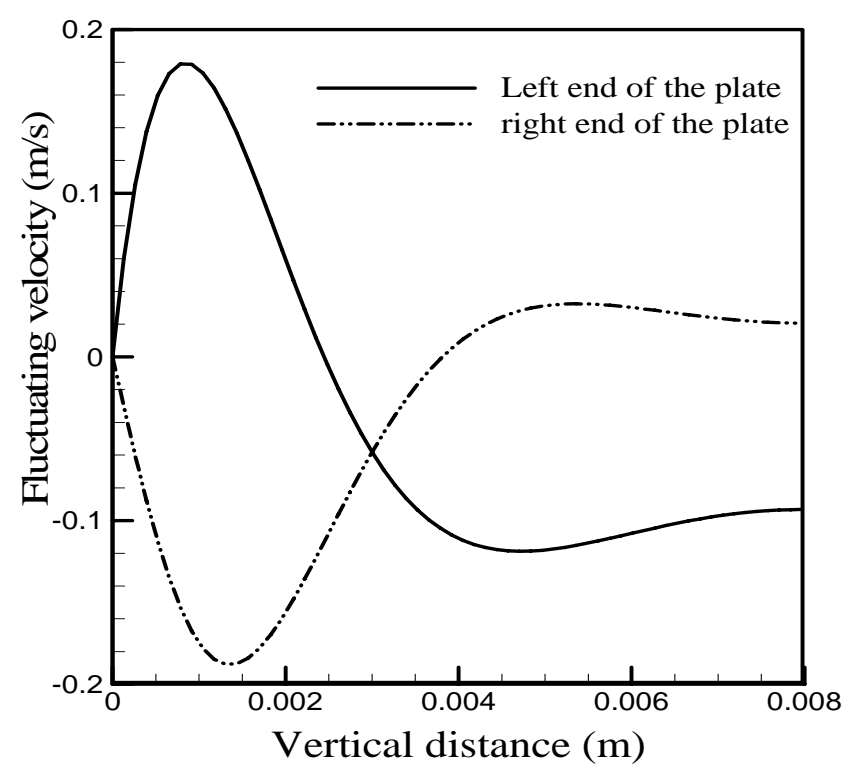

Fig. $12 X$-component of time averaged velocity vs $y$.

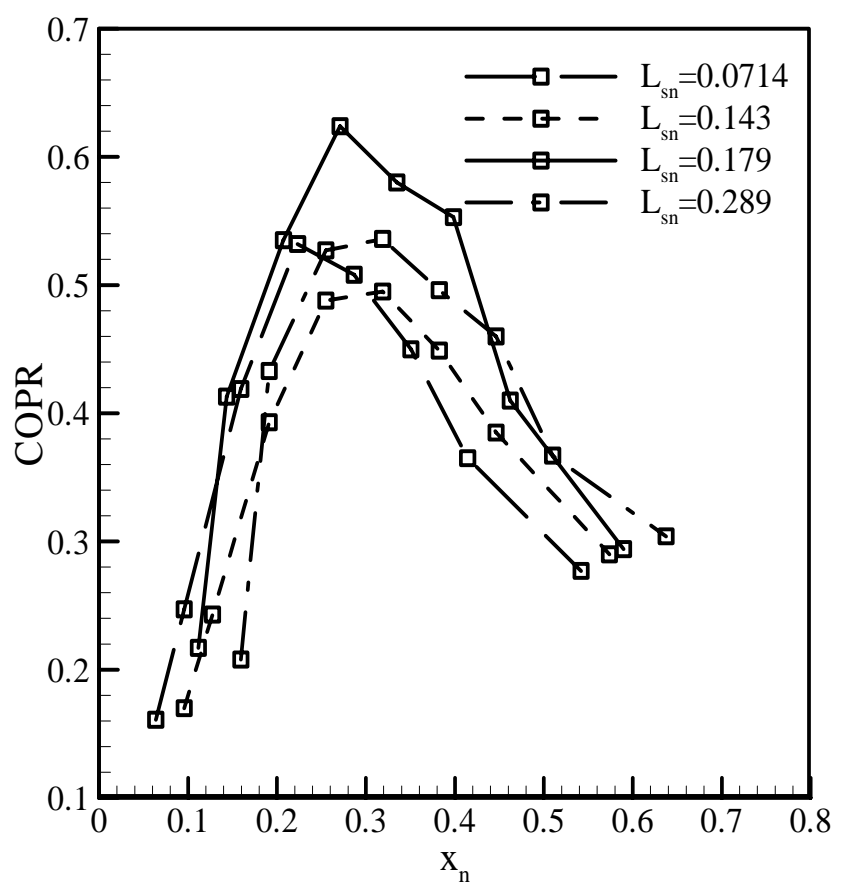

Fig. 13 COPR as a function of $x_{n}$ for different stack lengths.

thermoacoustic theory (Swift, 1988)) compared to the Carnot coefficient of performance) is presented in Fig. 13. Please refer to an article by Tasnim et al. (2010) for a detailed discussion and estimation of the COP (coefficient of performance) and COPR of a thermoacoustic heat pump. Stack center position and lengths are nondimensionalized in Fig. 13 using $x_{n}=k_{1} x_{c}$, where $k_{1}$ is the wave number ( $k_{1}=2 \pi / \lambda, \lambda$ is the wavelength of the standing wave) and $x_{c}$ is the stack center distance from the pressure anti-node, and $L_{s n}=k_{1} L, L$ is the length of the stack. The estimated COPR as a function of non-dimensionalized stack center position shows a parabolic behavior with a maximum for all the stacks considered, but the magnitude and the position of the peak changes as the stack length changes. The COPR shows a maximum at a stack length of 2.5 $\mathrm{cm}(0.025 \lambda)$ and a position of $4.25 \mathrm{~cm}$ which is almost $\approx \lambda / 20$ as suggested by Swift (2002). At this maximum location, COP of the stack is roughly $60 \%$ of Carnot's COP. The maximum COPR of a 
thermoacoustic refrigerator designed and tested by Hofler is 0.12 as indicated by Swift (1988). Note that for calculating the COPR, the author measured the COP experimentally as opposed to using linear theory in the present study. In the present study, due to the absence of heat exchangers, COP is calculated using the linear theory. The COPR indicated in the literature by Swift (1988) is for the complete thermoacoustic refrigerator as opposed to only stack in the present study. While considering the efficiency of a loudspeaker, and losses (viscous and thermal) along the stack, heat exchangers, and resonators the COPR of a complete thermoacoustic heat pump will be lower than the COPR of a stack as presented in the presented study.

\section{CONCLUSIONS}

Temperature oscillations within a stack of a thermoacoustic heat pump are measured experimentally. Some important information is revealed in the present study. First, axial heat transfer at the stack extremities exist. The temperature measurements at the extremities and at the neighboring gas show axial heat transfer at the stack extremities, as opposed to the hypothesis of a perfectly isolated stack used in the linear thermoacoustic theory. Four different mechanisms of heat transfer are identified at the stack extremities between the stack ends and the neighboring heat exchangers in the present study. High convective heat transfer is observed at the hot end of the stack compared to the cold end, because of the high temperature difference between the hot end and the corresponding gas. Second temperatures along the stack and the nearby gas show linear behavior. Temperature measurements along the stack reveal a linear behavior along the stack and the corresponding gas residing near the stacks. Third temperature gradient along the stack plate is less than that of along the working fluid. Therefore, accurate measurement of temperature difference along the stack using linear theory should be modified to take into account this difference of temperature gradient through the stack plate and the working fluid.

\section{REFERENCES}

Atchley, A. A., Hofler, T. J., Muzzerall, M. L., Kite, M. D., and Ao, C., 1990, "Acoustically generated temperature gradients in short plates,” Journal of the Acoustical Society of America, 88, 251-263. DOI: $10.1121 / 1.399947$

Brewster, J. R., Raspet, R., Bass, H. E., 1997, "Temperature discontinuities between elements of thermoacoustic devices,” Journal of the Acoustical Society of America, 102(6) 3355-3360.

DOI: $10.1121 / 1.419578$

Garrett, S. L., Perkins, D.K., Gopinath, A., 1994, "Thermoacoustic refrigerator heat exchangers: design, analysis and fabrication," in: G.F. Hewitt (Ed.), Proceedings of the Tenth International Heat Transfer Conference, Brighton, UK, 375-380.

Gusev, V., Lotton, P., Bailliet, H., Job, S., and Bruneau, M., 2001, "Thermal wave harmonics generation in the hydrodynamic heat transport in thermoacoustics," Journal of the Acoustical Society of America, 109, 84-90,

DOI:10.1121/1.332383

Lotton, P., Blanc-Benon, Ph., Bruneau, M., Gusev, V., Duffourd, S., Mironov, M., and Poignand, G., 2009, “Transient temperature profile inside thermoacoustic refrigerators, International Journal of Heat and Mass Transfer,” 52, 4986-4996.
DOI: 10.1016/j.ijheatmasstransfer.2009.03.075.

Marx, D., and Blanc-Benon, Ph., 2004, "Numerical simulation of stack-heat exchangers coupling in a thermoacoustic refrigerator," AIAA Journal, 42, 1338-1346.

DOI:10.2514/1.4342.

Marx, D, and Blanc-Benon, Ph., 2005, "Numerical calculations of the temperature difference between the extremities of a thermoacoustic stack plate," Cryogenics, 45, 163-172.

DOI: 10.1016/j.cryogenics.2004.08.007

Mozurkewich, G., 2001, "Heat transfer from transverse tubes adjacent to a thermoacoustic stack," Journal of the Acoustical Society of America, 110(2), 841-847.

DOI: $10.1121 / 1.385180$

Piccolo, A, and Cannistraro, G., 2002, "Convective heat transport along a thermoacoustic couple in the transient regime," International Journal Thermal Sciences, 41, 1067-1075. DOI: 10.1016/S1290-0729(02)01393-5

Poignand, G., Lihoreau, B., Lotton, P., Gaviot, E., Bruneau, M., Gusev, V., 2007, "Optimal acoustic fields in compact thermoacoustic refrigerators,” Applied Acoustics, 68, 642-659.

DOI: 10.1016/j.apacoust.2006.03.009.

Reid, R. S., Swift, G. W., 2000, "Experiments with a flow-through thermoacoustic refrigerator," Journal of the Acoustical Society of America, 108(6), 2835-2842.

DOI: $10.1121 / 1.1323721$.

Swift, G.W., 2002, "Thermoacoustics: A unifying perspective for some engines and refrigerators," Condensed Matter and Thermal Physics Group, Los Alamos National Laboratory, LA-UR 99-895.

Swift, G.W., 1992, "Analysis and performance of a large thermoacoustic engine," Journal of the Acoustical Society of America, 92(3), 1551-1563. DOI: $10.1121 / 1.403896$

Swift, G. W., 1988, "Thermoacoustic engines," Journal of the Acoustical Society of America, 84, 1145-1180. DOI: 10.1121/1.396617.

Tasnim, S.H., and Fraser, R.A., 2010, "Compressible pulsating convection through regular and random porous media: The thermoacoustic case," J. Heat Mass Transfer, submission reference no. HMT-10-0394.

Tasnim, S. H., 2009, "Transport phenomena in thermoacoustic engine," a report submitted to the examination committee for the partial fulfillment of the thesis requirement for the degree of doctor of philosophy in University of Waterloo, Canada, June 17.

Worlikar, A. S., Knio, O. M., and Klein, R., 1998, "Numerical simulation of a thermoacoustic refrigerator: Stratified flow around the stack,” Journal of Computational Physics, 144, 299-324.

DOI:10.1006/jcph.1997.5816 